\title{
Video Article \\ Adsorption Device Based on a Langatate Crystal Microbalance for High Temperature High Pressure Gas Adsorption in Zeolite H-ZSM-5
}

\author{
Wenjin Ding ${ }^{1}$, Giulia Baracchini ${ }^{1}$, Michael Klumpp ${ }^{2}$, Wilhelm Schwieger ${ }^{2}$, Roland Dittmeyer ${ }^{1}$ \\ ${ }^{1}$ Institute for Micro Process Engineering, Karlsruhe Institute of Technology (KIT) \\ ${ }^{2}$ Institute of Chemical Reaction Engineering, University Erlangen-Nürnberg (FAU)
}

Correspondence to: Wenjin Ding at info@imvt.kit.edu

URL: https://www.jove.com/video/54413

DOI: doi: $10.3791 / 54413$

Keywords: Engineering, Issue 114, high-frequency oscillating microbalance, high-temperature gas adsorption, Langmuir isotherm, microporous materials, steam-assisted crystallization, zeolite coating, dimethyl ether synthesis

Date Published: 8/25/2016

Citation: Ding, W., Baracchini, G., Klumpp, M., Schwieger, W., Dittmeyer, R. Adsorption Device Based on a Langatate Crystal Microbalance for High Temperature High Pressure Gas Adsorption in Zeolite H-ZSM-5. J. Vis. Exp. (114), e54413, doi:10.3791/54413 (2016).

\section{Abstract}

We present a high-temperature and high-pressure gas adsorption measurement device based on a high-frequency oscillating microbalance ( $5 \mathrm{MHz}$ langatate crystal microbalance, LCM) and its use for gas adsorption measurements in zeolite H-ZSM-5. Prior to the adsorption measurements, zeolite H-ZSM- 5 crystals were synthesized on the gold electrode in the center of the LCM, without covering the connection points of the gold electrodes to the oscillator, by the steam-assisted crystallization (SAC) method, so that the zeolite crystals remain attached to the oscillating microbalance while keeping good electroconductivity of the LCM during the adsorption measurements. Compared to a conventional quartz crystal microbalance (QCM) which is limited to temperatures below $80^{\circ} \mathrm{C}$, the LCM can realize the adsorption measurements in principle at temperatures as high as $200-300^{\circ} \mathrm{C}$ (i.e., at or close to the reaction temperature of the target application of onestage DME synthesis from the synthesis gas), owing to the absence of crystalline-phase transitions up to its melting point $\left(1,470{ }^{\circ} \mathrm{C}\right)$. The system was applied to investigate the adsorption of $\mathrm{CO}_{2}, \mathrm{H}_{2} \mathrm{O}$, methanol and dimethyl ether (DME), each in the gas phase, on zeolite $\mathrm{H}-\mathrm{ZSM}-5$ in the temperature and pressure range of $50-150{ }^{\circ} \mathrm{C}$ and $0-18$ bar, respectively. The results showed that the adsorption isotherms of these gases in $\mathrm{H}$ ZSM-5 can be well fitted by Langmuir-type adsorption isotherms. Furthermore, the determined adsorption parameters, i.e., adsorption capacities, adsorption enthalpies, and adsorption entropies, compare well to literature data. In this work, the results for $\mathrm{CO}_{2}$ are shown as an example.

\section{Video Link}

The video component of this article can be found at https://www.jove.com/video/54413/

\section{Introduction}

Adsorption properties strongly influence the performance of catalytic materials, hence precise knowledge of these properties can assist in characterization, design and optimization of such materials. However, the adsorption properties are generally judged from single-component adsorption measurements often at room temperatures or even under liquid nitrogen conditions, and therefore an extension to practical situations may lead to a severe deviation from the real behavior. In situ adsorption measurements on catalytic materials, especially at high temperature and high pressure conditions, still remain a great challenge.

An adsorption measurement device based on a quartz crystal microbalance (QCM) is advantageous over the commercialized volumetric and gravimetric methods in a way that it is highly accurate for mass sorption applications, satisfactorily stable in a controlled environment, and more affordable ${ }^{1-2}$. However, the conventional QCM analysis is limited to temperatures below $80^{\circ} \mathrm{C}^{1-2}$. In order to overcome this limitation, we developed an adsorption measurement device based on a high-temperature high-frequency oscillating microbalance (langatate crystal microbalance, $\mathrm{LCM})^{3}$, which can realize the adsorption measurements in principle at temperatures as high as $200-300{ }^{\circ} \mathrm{C}$, owing to the absence of crystalline-phase transitions up to its melting point $\left(1,470{ }^{\circ} \mathrm{C}\right)^{4}$. The LCMs used in this work have an AT-cut (i.e., the plate of the crystal microbalance contains the $x$ axis of the crystal and is inclined by $35^{\circ} 15^{\prime}$ from the $z$ axis) and a resonant frequency of $5 \mathrm{MHz}$. This device was applied to the adsorption measurements of $\mathrm{CO}_{2}, \mathrm{H}_{2} \mathrm{O}$, methanol, and dimethyl ether (DME), each in gaseous state, on zeolite $\mathrm{H}-\mathrm{ZSM}-5$ in the temperature range of $50-150{ }^{\circ} \mathrm{C}$ and pressure range of $0-18 \mathrm{bar}^{3}$, aiming at the validation of simulation models for the optimization of bifunctional core-shell catalysts for one-stage production of DME from the synthesis gas ${ }^{5-6}$. How to operate this device for gas adsorption measurements is presented in the protocol section.

Prior to the adsorption measurements, zeolite H-ZSM- 5 crystallites $(0.502 \mathrm{mg})$ were synthesized on the gold electrode in the center of the LCM by the steam-assisted crystallization (SAC) method according to de la Iglesia et al. ${ }^{7}$, in such a way that the zeolite crystallites remain attached to the oscillating microbalance. As shown in Figure 1, the LCM used in the adsorption measurement device has polished gold electrodes on both sides, which help to connect the LCM to an oscillator. Since the zeolite crystals on the connection points of the gold electrodes to the oscillator would significantly reduce the electroconductivity (as indicated in Figure 1) and thus the measurement sensitivity of the LCM, the zeolite H- 
ZSM- 5 crystals were deposited on the LCM via the SAC method not covering these connection points ${ }^{3}$. The details about the synthesis of zeolite H-ZSM-5 on LCM are briefly summarized in the following protocol section and shown in the video protocol in detail.

Protocol

Caution: Please consult all relevant material safety data sheets (MSDS) before use. Several of the chemicals used in the synthesis of zeolite H-ZSM-5 are acutely toxic and carcinogenic. Nanomaterials may have additional risks compared to their bulk counterpart. Please use all appropriate safety practices when performing a nanocrystal reaction including the use of engineering controls (fume hood, glovebox) and personal protective equipment (safety glasses, gloves, lab coat, full length pants, closed-toe shoes). Moreover, pay special attention when, performing the adsorption measurements with methanol and DME, since both are flammable and explosive dangerous materials.

\section{Synthesis of Zeolite H-ZSM-5 on the LCM}

1. Preparation of a zeolite synthesis mixture

Note: The final synthesis mixture had the following molar composition as adapted from de la Iglesia et al. ${ }^{7}: 1 \mathrm{SiO}_{2}: 50 \mathrm{H}_{2} \mathrm{O}: 0.07 \mathrm{Na}_{2} \mathrm{O}$ :

$0.024 \mathrm{TPA}_{2} \mathrm{O}: 0.005 \mathrm{Al}_{2} \mathrm{O}_{3}$. Thus, the theoretical Si/Al molar ratio of the synthesized zeolite $\mathrm{H}-\mathrm{ZSM}-5$ is 100.

1. Dissolve $0.14 \mathrm{~g}$ sodium hydroxide in $20.30 \mathrm{~g}$ de-ionized water via stirring. Alternatively, mix $3.64 \mathrm{~g}$ of $1 \mathrm{M} \mathrm{NaOH}$ with $16.8 \mathrm{~g}$ de-ionized water.

2. Add $1.16 \mathrm{~g}$ tetrapropylammonium hydroxide (TPAOH) solution, and stir the solution until it appears clear.

3. Add $5.0 \mathrm{~g}$ tetraethyl orthosilicate (TEOS) solution dropwise, and stir the solution until it appears clear.

4. Keep stirring, while adding $0.09 \mathrm{~g}$ aluminum nitrate nonahydrate $\left(\mathrm{Al}\left(\mathrm{NO}_{3}\right)_{3} \cdot 9 \mathrm{H}_{2} \mathrm{O}\right.$, solid) into the solution.

5. Keep stirring until the solid aluminum nitrate nonahydrate is dissolved. Note that the prepared zeolite synthesis mixture should be used within $5 \mathrm{hr}$ due to its aging.

2. Synthesis of zeolite ZSM-5 on the LCM via SAC ${ }^{3}$

1. Clean the LCM before the zeolite synthesis

1. Wash the LCM thoroughly with the de-ionized water.

2. Put the LCM in a beaker with the de-ionized water, and clean it in an ultrasound bath

3. Dry the LCM at $80{ }^{\circ} \mathrm{C}$ in an oven.

\section{Zeolite synthesis}

1. Carefully place several drops of the prepared zeolite synthesis mixture on the electrode in the center of the LCM as shown in Figure 1 using a pipette, since only the zeolite deposited on the gold electrode can cause the resonant frequency shift of the $\mathrm{LCM}^{8}$. Moreover, avoid spreading the synthesis mixture on the connection points of the gold electrodes to the oscillator, since the zeolite on the connection points would significantly reduce the electroconductivity and thus the measurement sensitivity of the LCM. In addition, removing the zeolite crystals on the connection points after the deposition will destroy the electrodes.

2. Dry the LCM with the synthesis mixture at $80^{\circ} \mathrm{C}$ for $2 \mathrm{hr}$ to obtain a highly viscous gel-like phase on it.

3. Add a small amount of de-ionized water (about $10 \mathrm{ml}$ ) in a Teflon-lined autoclave $(80 \mathrm{ml}$ ) in order to produce the steam during the zeolite synthesis.

4. Put the Teflon holder in the autoclave, which supports the LCM horizontally above the liquid water at the bottom of the autoclave during the zeolite synthesis.

5. Keep the autoclave in an oven at $150{ }^{\circ} \mathrm{C}$ for $48 \mathrm{hr}$ to synthesize the zeolite on the LCM via the SAC method.

6. Right after the SAC, wash the coated LCM with the de-ionized water and dry it at $80^{\circ} \mathrm{C}$ for $2 \mathrm{hr}$.

7. Remove the organic template in the zeolite crystals by calcination in a high-temperature oven under an oxidative atmosphere. Program the oven as follows: a) Increase the temperature from ambient to $450{ }^{\circ} \mathrm{C}$ at a rate of $3^{\circ} \mathrm{C} \min ^{-1}$; b) Keep the temperature at $450{ }^{\circ} \mathrm{C}$ for $4 \mathrm{hr}$; c) Decrease the temperature from $450{ }^{\circ} \mathrm{C}$ to room temperature at a rate of $3^{\circ} \mathrm{C} \mathrm{min}{ }^{-1}$.

8. Dissolve $26.75 \mathrm{~g}$ ammonium chloride $\left(\mathrm{NH}_{4} \mathrm{Cl}\right.$, solid) in $0.4 \mathrm{~L}$ de-ionized water. Add more de-ionized water in the solution so that the final $\mathrm{NH}_{4} \mathrm{Cl}$ solution is $0.5 \mathrm{~L}$ and has the concentration of $1 \mathrm{~mol} \mathrm{dm}^{-3}$.

9. Put the coated LCM into the $\mathrm{NH}_{4} \mathrm{Cl}$ solution $(0.2 \mathrm{~L})$ in a beaker, and ion-exchange the $\mathrm{Na}-\mathrm{ZSM}-5$ crystals coated on the LCM at $20{ }^{\circ} \mathrm{C}$ for $2 \mathrm{hr}$. Repeat the ion-exchange using $0.2 \mathrm{~L}$ fresh $\mathrm{NH}_{4} \mathrm{Cl}$ solution to get the $\mathrm{NH}_{4}-\mathrm{ZSM}-5$ crystals.

10. Obtain the H-ZSM-5 by final calcination using the same parameters as mentioned in Step 1.2.2.7.

\section{Adsorption Measurements Using the LCM-based Adsorption Measurement Device ${ }^{3}$}

Note: In this work, the LCM without coating and the one coated with H-ZSM-5 (prepared in the last section) is termed "reference LCM" and "sample LCM", respectively. Moreover, the sample LCM before zeolite deposition is termed "unloaded sample LCM". In a previous publication in Journal of Physical Chemistry $C^{3}$, a detailed description of the LCM-based adsorption measurement device can be found. In this work, the operation of the device for gas adsorption measurements is presented in this short protocol and in the video protocol in detail.

1. Preparation before adsorption measurements

1. Tests on the effects of temperature and pressure on the difference in resonant frequencies of the reference and unloaded sample LCMs

1. Clean the O-ring, the LCM holder, and the sample chamber with acetone and pressed air.

2. Put the reference and unloaded sample LCMs in a beaker with de-ionized water and clean them in an ultrasound bath.

3. Carefully place the clean reference and unloaded sample LCMs on the LCM holder, which is connected to the oscillator via high temperature resistant electric cables. 
4. Pretest the installed LCMs using the oscillator to ensure that the resonant frequencies can be detected successfully.

5. Close the sample chamber, and evacuate it by a vacuum pump.

6. Change the pressure in the sample chamber via dosing pure $\mathrm{N}_{2}$.

7. Control the temperature inside the sample chamber by a temperature controller.

8. Measure the resonant frequencies of the reference and unloaded sample LCMs in the studied temperature and pressure ranges, i.e., $50-150{ }^{\circ} \mathrm{C}$ and $0-16$ bar, in order to know the effect of temperature and pressure on the difference in resonant frequencies of the reference and unloaded sample LCMs $\left(\Delta f_{0}\right.$ in Step 2.2.4). The tests show that $\Delta f_{0}$ is significantly affected by the temperature $\left(1,200\right.$ to $3,000 \mathrm{~Hz}$ at $50-150^{\circ} \mathrm{C}$ ), whereas the gas pressure has no significant effect (change of $\Delta f_{0}$ smaller than $300 \mathrm{~Hz}$ in the pressure range of 0-16 bar). Use the determined values of $\Delta f_{0}$ in the Sauerbrey equation in Step 2.2.4 to calculate the adsorbed amount of gases on the zeolite.

\section{Activation of sample LCM}

1. Clean the O-ring, the LCM holder, and the sample chamber with acetone and pressed air.

2. Put the reference LCM in a beaker with de-ionized water, and clean it in an ultrasound bath.

3. Carefully place the clean reference LCM and sample LCM on the LCM holder, which is connected to the oscillator via high temperature resistant electric cables.

4. Pretest the placed LCMs using the oscillator to ensure that the resonant frequencies can be detected successfully.

5. Close the sample chamber, and evacuate it by a vacuum pump.

6. Activate the sample LCM at high temperatures (at least $50^{\circ} \mathrm{C}$ higher than the temperatures of the adsorption measurements, $200{ }^{\circ} \mathrm{C}$ in this work) in vacuum condition overnight to ensure that only a negligible gas amount is adsorbed on the $\mathrm{H}-\mathrm{ZSM}-5$.

\section{Adsorption measurements}

Note: In this work, the adsorption measurement of $\mathrm{CO}_{2}$ at $50{ }^{\circ} \mathrm{C}$ is presented to give an example. The obtained data from the measurement (e.g., resonant frequencies) and the calculated masses of adsorbed $\mathrm{CO}_{2}$ on the H-ZSM-5 can be found in Table S1 of the Supporting Information of our previous publication ${ }^{3}$.

1. Adjust the temperature inside the sample chamber at the desired temperature of the adsorption measurements $\left(i . e ., 50 \pm 0.1^{\circ} \mathrm{C}\right)$ by a temperature controller, under vacuum conditions, i.e., only with a negligible amount of adsorbed gas.

2. Connect the oscillator to the sample LCM, and measure its resonant frequency by the supporting software of the oscillator via fitting the experimental data with a Butterworth-Van Dyke equivalent circuit model.

3. Switch the connection of the oscillator to the reference LCM, and measure its resonant frequency.

4. Use the measured resonant frequencies of the sample and reference LCMs under vacuum conditions to determine the mass of $\mathrm{H}$ ZSM-5 deposited on the sample LCM (without adsorbed gas) according to the Sauerbrey equation ${ }^{2,8}$ :

$$
\Delta m=\frac{n \cdot\left(\Delta f-\Delta f_{0}\right) \cdot \sqrt{\rho_{L} \cdot \mu_{L}}}{2 f_{0, r}{ }^{2}} \cdot A
$$

where $\Delta m$ is the difference in mass in $\mathrm{g}, n$ is the number of the harmonic at which the crystal is driven (in this study, $n=3$ ), $\Delta f$ is the difference in resonant frequencies of reference and sample LCMs in $\mathrm{Hz}, \Delta f_{0}$ is the difference of the resonant frequencies between the reference and unloaded sample LCM in $\mathrm{Hz}, \rho_{L}$ is the density of the langatate crystal $\left(6.13 \mathrm{~g} \mathrm{~cm}^{-3}\right)^{4}, \mu_{L}$ is the effective piezoelectrically stiffened shear modulus of the langatate crystal $\left(1.9 \times 10^{12} \mathrm{~g} \mathrm{~cm}^{-1} \mathrm{sec}^{-2}\right)^{4}, f_{0}$, is the resonant frequency of the reference LCM, i.e., the unloaded LCM, $A$, the area of the LCM $\left(1.539 \mathrm{~cm}^{2}\right)^{3}$.

Note: In this work, the mass of H-ZSM- 5 deposited on the gold electrode in the center of the LCM is $0.502 \mathrm{mg}$, which causes a resonant frequency shift of $14,100 \mathrm{~Hz}$ at $50^{\circ} \mathrm{C}$.

5. Control the gas pressure of $\mathrm{CO}_{2}$ inside the sample chamber by dosing pure gas from the gas cylinder via a mass flow controller (for methanol and DME, from the evaporator manually via a dosing valve into the chamber), or by evacuation via a vacuum pump. Here, use a pressure range of $\mathrm{CO}_{2}$ adsorption measurements of 0-16 bar as shown in Figure 2.

6. Wait until equilibrium conditions and a stable temperature have been reached, e.g., the temperature varies within $50 \pm 0.1^{\circ} \mathrm{C}$.

7. Connect the oscillator to the sample LCM, and measure its resonant frequency after exposure to the gas at a given pressure.

8. Switch the connection of the oscillator to the reference LCM, and measure its resonant frequency under the same conditions.

9. According to the Sauerbrey equation shown above, calculate the total mass of H-ZSM-5 deposited on the sample LCM and gas adsorbed on the H-ZSM-5 under this gas pressure. By subtracting the mass of H-ZSM-5 (without adsorbed gas) determined in Step 2.2.4, the mass of $\mathrm{CO}_{2}$ adsorbed on the $\mathrm{H}-\mathrm{ZSM}-5$ under this gas pressure is obtained.

10. Repeat the resonant frequency measurements for sample and reference LCMs for varying pressures, in order to obtain all the masses of $\mathrm{CO}_{2}$ adsorbed on the $\mathrm{H}-\mathrm{ZSM}-5$ sample under different gas pressures.

11. Finally, obtain the gas adsorption isotherm at $50{ }^{\circ} \mathrm{C}$ in the studied pressure range of $0-16$ bar via calculating all the masses of $\mathrm{CO}_{2}$ adsorbed on the H-ZSM-5 sample under different gas pressures according to Step 2.2.9.

12. For adsorption isotherms at other temperatures, change the stable temperature using the temperature controller, and repeat Steps 2.2.1 to 2.2.11.

13. Fit the adsorption isotherms with adsorption models like Langmuir models via the least squares method to determine the adsorption parameters like adsorption capacities, adsorption enthalpies, and adsorption entropies (see previous publication ${ }^{3}$ and its Supporting Information). 


\section{Representative Results}

Figure 1 shows the photographs, light microscopy and scanning electron microscopy (SEM) images of the coated and uncoated LCM sensor (left), as well as their X-ray diffraction (XRD) patterns (right). From both, light and scanning electron microscopy (Figure 1b and c), the connection points of the gold electrodes to the oscillator are less covered with zeolite crystals than the center region of the LCM. Most of the zeolite crystals on top of the LCM-sensor are isolated and show characteristic rounded-boat morphology, with the (010)-plane predominantly facing up. Besides, some crystals additionally show the typical intergrowth behavior ("twinned crystals"). Moreover, the loaded H-ZSM-5 (Si/Al molar ratio of 100 according to the composition of the synthesis mixture) on the langatate crystal has been investigated by XRD and wavelengthdispersive X-ray (WDX) spectroscopy ${ }^{3}$.

In Figure 2, $\mathrm{CO}_{2}$ adsorption isotherms for the $\mathrm{H}-\mathrm{ZSM}-5$ zeolite obtained with the LCM device in the temperature range of $50-150{ }^{\circ} \mathrm{C}$ and pressure range of $0-16 \mathrm{bar}$, as well as the fit of the single site Langmuir isotherm model to the experimental data, are shown to give a representative example. As shown in Figure 2, the determined adsorption isotherms of $\mathrm{CO}_{2}$ were fitted with a single site Langmuir isotherm well. Figure 3 shows the diagram of $\ln \left(K_{i}^{\prime}\right)$ vs. 1,000/T for $\mathrm{CO}_{2}$ as derived from the adsorption isotherms, i.e., the temperature dependence of the adsorption constants determined from the fit of the adsorption isotherms. The adsorption enthalpies and entropies of $\mathrm{CO}_{2}$ were determined by fitting with the van't Hoff equation (see the Supporting Information of the previous publication ${ }^{3}$ ). The results of the model fitting show that the adsorption capacity, adsorption enthalpy and adsorption entropy for $\mathrm{CO}_{2}$ in $\mathrm{H}-\mathrm{ZSM}-5$ are $4.0 \pm 0.2 \mathrm{mmol} \mathrm{g}^{-1}, 15.3 \pm 0.5 \mathrm{~kJ} \mathrm{~mol}^{-1}$ and $56.3 \pm 1.5 \mathrm{~J}$ $\mathrm{mol}^{-1} \mathrm{~K}^{-1}$, respectively ${ }^{3}$.

The high quality of the fit of the single site Langmuir isotherm and the van't Hoff equation as shown in Figures 2 and $\mathbf{3}$ supports the assumption of a constant adsorption capacity (i.e., saturation loading) and enthalpy (i.e., heat of adsorption) to be valid at least for the range of conditions used. Moreover, the adsorption parameters of $\mathrm{CO}_{2}$ determined by the LCM-based adsorption measurement device in this work compare well to values reported in literature ${ }^{9-12}$, i.e., the adsorption capacity, adsorption enthalpy and adsorption entropy reported for $\mathrm{CO}_{2}$ in $\mathrm{MFI}$-type zeolites vary in the range of 2.1-3.8 $\mathrm{mmol} \mathrm{g}^{-1}, 19-28.7 \mathrm{~kJ} \mathrm{~mol}^{-1}$, and $43.7-82.7 \mathrm{~J} \mathrm{~mol}^{-1} \mathrm{~K}^{-1}$, respectively, in the temperature range of $30-200{ }^{\circ} \mathrm{C}$ and pressure range of $0-5$ bar.
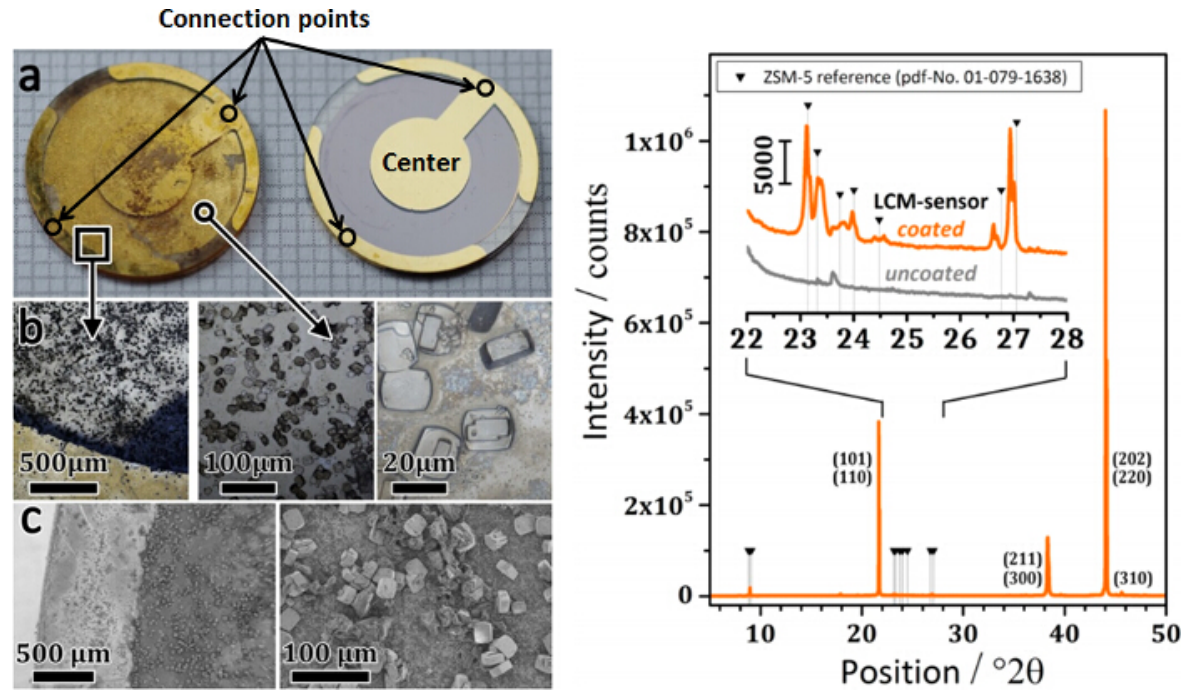

Figure 1. Coated langatate crystal microbalance sensor (left). (a) Photographs of the coated and uncoated sensor (right), (b) light microscopy and (c) scanning electron microscopy images. The X-ray diffraction patterns of coated and uncoated LCM sensor (right). This figure has been modified from a previous publication ${ }^{3}$. Reprinted with the permission of American Chemical Society (Copyright 2015). Please click here to view a larger version of this figure. 


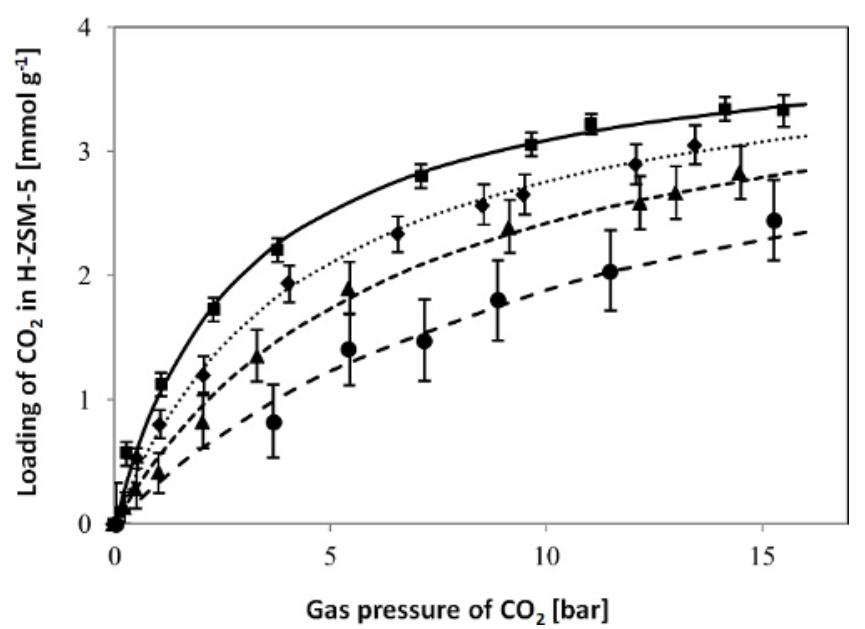

Figure 2. Adsorption isotherms for $\mathrm{CO}_{2}$ in $\mathrm{H}-\mathrm{ZSM}-5$ at $50(\square), 75(\bullet), 100(\Delta)$, and $150{ }^{\circ} \mathrm{C}(\bullet)$. The symbols represent the experimental data, the error bars indicate the measurement uncertainty of the resonant frequencies caused by, e.g., the temperature instability, and calculated according to the Sauerbrey equation as described in Step 2.2.4, and the lines represent the fit of the single site Langmuir isotherm model to the experimental data. This figure has been modified from a previous publication ${ }^{3}$. Reprinted with the permission of American Chemical Society (Copyright 2015). Please click here to view a larger version of this figure.

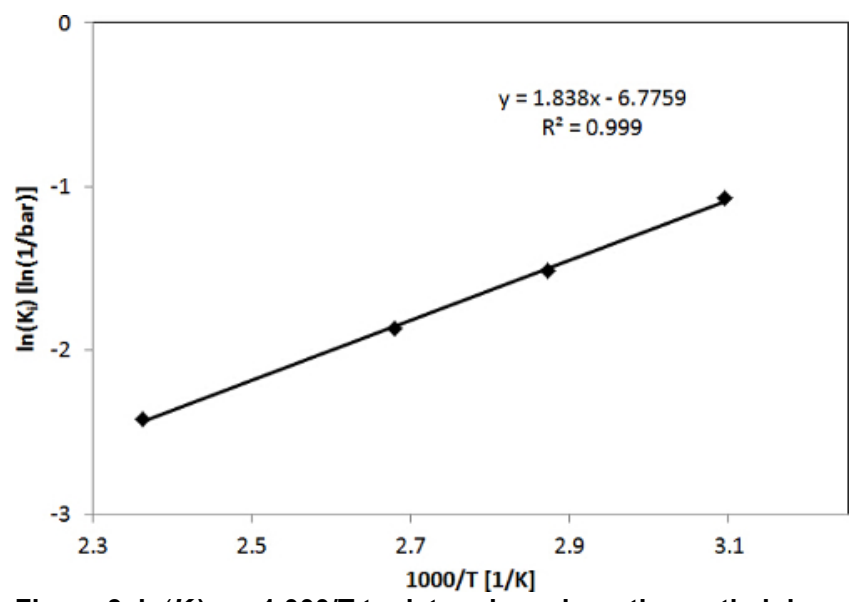

Figure 3. $\ln \left(K_{i}\right)$ vs. $1,000 / T$ to determine adsorption enthalpies and entropies for $\mathrm{CO}_{2}$. This figure has been modified from a previous publication ${ }^{3}$. Reprinted with the permission of American Chemical Society (Copyright 2015). Please click here to view a larger version of this figure.

\section{Discussion}

In this work, the successful synthesis of the zeolite H-ZSM-5 crystals on the gold electrode in the center of the LCM sensor by SAC is demonstrated, i.e., the zeolite is successfully loaded on the LCM sensor without covering the connection points of the gold electrodes to the oscillator. Thus, the zeolite can oscillate together with the LCM sensor, while the LCM sensor keeps its good electroconductivity and measurement sensitivity. Compared to the conventional QCM devices which are limited below $80^{\circ} \mathrm{C}$, the LCM device presented in this work is successfully used for the adsorption measurements at temperatures as high as $150^{\circ} \mathrm{C}$, i.e., at or close to the temperature of reactions in industry. However, the present LCM device is limited below $200^{\circ} \mathrm{C}$. At temperatures higher than $200^{\circ} \mathrm{C}$, the measurement uncertainty may exceed the mass of the adsorbed gas, since, with the increasing temperature above $150{ }^{\circ} \mathrm{C}$, the mass of the adsorbed gas has a significant decrease, whereas the measurement uncertainty increases significantly due to the decreasing temperature control precision. Thus, in future experiments, a new method should be developed to deposit more zeolite on the LCM, which causes more gas to adsorb, and moreover compensates the effect of the temperature and pressure on $\Delta f_{0}$. This could help to extend the application range of the LCM device to higher temperatures.

During the experiment, the critical steps in the zeolite synthesis are Steps 1.2.2.1, 1.2.2.4, 1.2.2.5 and 1.2.2.7, while those in the adsorption measurements are Steps 2.1.1.3, 2.1.1.4, 2.2.1, 2.2.5 and 2.2.6. In Step 1.2.2.1, avoid placing too much of the synthesis mixture on the LCM, which would spread on the connection points of the gold electrodes. In Step 1.2.2.4, carefully put the Teflon holder with the LCM in the autoclave to ensure that the LCM is horizontal and does not contact the liquid water at the bottom. In Steps 1.2.2.5 and 1.2.2.7, do not use a higher temperature in the zeolite synthesis and calcination, since our previous experiments show that it leads to degradation of the LCM. In the adsorption measurements, the position of the LCM sensors has a significant effect on the connectivity of the LCM sensors to the oscillator, and therefore on the quality of the resonant frequency signals. Therefore, pay special attention to Steps 2.1.1.3 and 2.1.1.4, in which the LCMs are loaded on the holder and pretested. The LCMs should be in the position that they are connected with the oscillator via the connection points of the electrodes (indicated in Figure 1). This is mandatory to obtain high quality resonant frequency signals enabling high measurement accuracy. 
In addition, in Steps 2.2.1 and 2.2.6, ensure that a stable temperature is achieved before the measurements, since this also increases the measurement accuracy. Moreover, in Step 2.2.5, feed the gas slowly, in order to have a small change of the temperature inside. This helps the temperature to become stable again after a short time.

Since the SAC synthesis method for zeolite H-ZSM- 5 on the LCM sensor could be extended to other zeolites easily, the LCM-based adsorption measurement device is expected to be used for them as well. Moreover, due to its high accuracy and low cost, this device is expected to be applicable to any material, which could be coated on the LCM, in order to investigate its adsorption properties at high temperatures.

\section{Disclosures}

The authors have nothing to disclose.

\section{Acknowledgements}

This research has been funded by Deutsche Forschungsgemeinschaft (DFG) within the framework of the priority program 1570: porous media with defined pore system in process engineering - modeling, application, synthesis, under grant numbers DI 696/9-1 to -3 and SCHW $478 / 23-1$ to -3 .

\section{References}

1. Tsionsky, V., Gileadi, E. Use of the Quartz Crystal Microbalance for the Study of Adsorption from the Gas Phase. Langmuir. 10, 2830-2835 (1994).

2. Venkatasubramanian, A. et al. Gas Adsorption Characteristics of Metal-Organic Frameworks via Quartz Crystal Microbalance Techniques. J. Phys. Chem. C. 116, 15313-15321 (2012).

3. Ding, W. et al. Investigation of High-Temperature and High-Pressure Gas Adsorption in Zeolite H-ZSM-5 via Langatate Crystal Microbalance: $\mathrm{CO}_{2}, \mathrm{H}_{2} \mathrm{O}$, Methanol and Dimethyl Ether, J. Phys. Chem. C. 119, 23478-23485 (2015).

4. Davulis, P.M., Pereira da Cunha, M. High-Temperature Langatate Elastic Constants and Experimental Validation up to $900{ }^{\circ} \mathrm{C}$. IEEE Trans Ultrason Ferroelectr Freq Control. 57, 59-65 (2010).

5. Ding, W., Li, H., Pfeifer, P., Dittmeyer, R. Crystallite-Pore Network Model of Transport and Reaction of Multicomponent Gas Mixtures in Polycrystalline Microporous Media, Chem. Eng. J. 254, 545-558 (2014).

6. Ding, W. et al. Simulation of One-Stage Dimethyl Ether Synthesis over Core/Shell Catalyst in Tube Reactor, Chem Ing Tech. 87, 702-712 (2015).

7. de la Iglesia, O. et al. Preparation of Pt/ZSM-5 Films on Stainless Steel Microreactors. Catal. Today. 125, 2-10 (2007).

8. Sauerbrey, G., Verwendung von Schwingquartzen zur Wägung dünner Schichten und zur Mikrowägung. Zeitschrift für Physik. 155, 206-222 (1959).

9. Wirawan, S.K., Creaser, D. $\mathrm{CO}_{2}$ Adsorption on Silicalite-1 and Cation Exchanged ZSM-5 Zeolites Using a Step Change Response Method, Microporous Mesoporous Mater. 91, 196-205 (2006).

10. Choudhary, V. R., Mayadevi, S. Adsorption of Methane, Ethane, Ethylene, and Carbon Dioxide on High Silica Pentasil Zeolites and Zeolitelike Materials Using Gas Chromatography Pulse Technique, Sep. Sci. Technol. 28, 2197-2209 (1993).

11. Choudhary, V. R., Mayadevi, S. Adsorption of Methane, Ethane, Ethylene, and Carbon Dioxide on Silicalite-I, Zeolites. 17, 501-507 (1996)

12. Zhu, W.D., Hrabanek, P., Gora, L., Kapteijn, F., and Moulijn, J.A. Role of Adsorption in the Permeation of $\mathrm{CH}_{4}$ and $\mathrm{CO}_{2}$ through a Silicalite-1 Membrane, Ind. Eng. Chem. Res. 45, 767-776 (2006). 